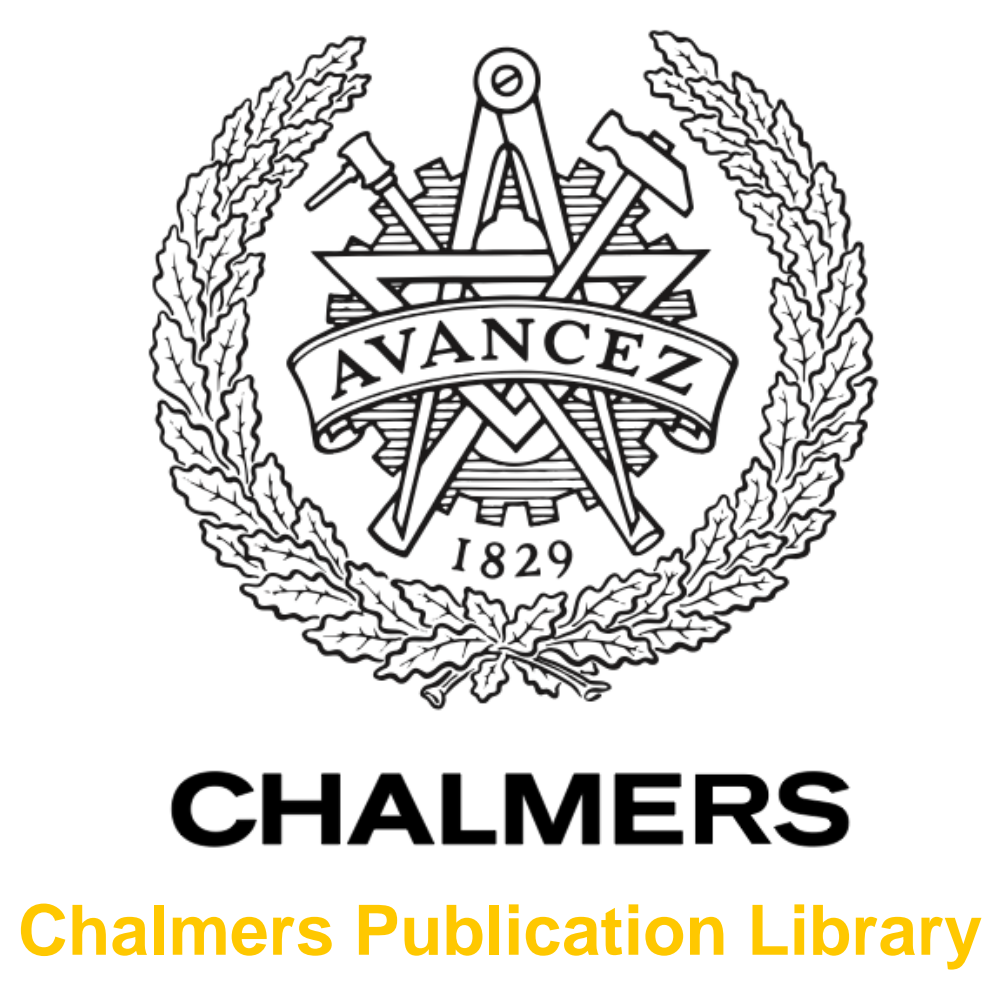

Robust adaptive beamforming for MIMO monopulse radar

This document has been downloaded from Chalmers Publication Library (CPL). It is the author's version of a work that was accepted for publication in:

Proceedings of SPIE - The International Society for Optical Engineering (ISSN: 0277786X)

Citation for the published paper:

Rowe, W. ; Ström, M. ; Li, J. (2013) "Robust adaptive beamforming for MIMO monopulse radar". Proceedings of SPIE - The International Society for Optical Engineering, vol. 8714

http://dx.doi.org/10.1117/12.2015805

Downloaded from: http://publications.lib.chalmers.se/publication/186987

Notice: Changes introduced as a result of publishing processes such as copy-editing and formatting may not be reflected in this document. For a definitive version of this work, please refer to the published source. Please note that access to the published version might require a subscription.

Chalmers Publication Library (CPL) offers the possibility of retrieving research publications produced at Chalmers University of Technology. It covers all types of publications: articles, dissertations, licentiate theses, masters theses, conference papers, reports etc. Since 2006 it is the official tool for Chalmers official publication statistics. To ensure that Chalmers research results are disseminated as widely as possible, an Open Access Policy has been adopted.

The CPL service is administrated and maintained by Chalmers Library. 


\title{
Robust adaptive beamforming for MIMO monopulse radar
}

\author{
William Rowe, Marie Ström, Jian Li and Petre Stoica *
}

\begin{abstract}
Researchers have recently proposed a widely separated multiple-input multiple-output (MIMO) radar using monopulse angle estimation techniques for target tracking. The widely separated antennas provide improved tracking performance by mitigating complex target radar cross-section fades and angle scintillation. An adaptive array is necessary in this paradigm because the direct path from any transmitter could act as a jammer at a receiver. When the target-free covariance matrix is not available, it is critical to include robustness into the adaptive beamformer weights. This work explores methods of robust adaptive monopulse beamforming techniques for MIMO tracking radar.
\end{abstract}

\section{INTRODUCTION}

Widely separated multiple-input multiple-output (MIMO) radar systems are of significant interest for tracking applications because of their ability to mitigate angular glint. The majority of real world targets act as complex scatters rather than ideal scatters which introduces the so-called target noise into the problem. ${ }^{1,2}$ This noise can manifest itself as amplitude and angle scintillations. The angle scintillations, commonly referred to as glint, is of great concern in tracking radar because it can cause the angle estimate to move well beyond the actual true target angle. ${ }^{3}$ The typical monopulse angle estimation techniques (or sequential lobing techniques) cannot account for glint due to the physical nature of the phenomenon which can be viewed as phase-front distortion. ${ }^{4,5}$

The best technique for accounting for glint has long been known to be to mitigate the physical phenomenon in the illumination scheme. ${ }^{4,6}$ The two methods of glint mitigation proposed have been frequency diversity and angle diversity since target glint has been shown to have a strong negative correlation with radar cross-section (RCS) nulls or fades. Since RCS is a function of carrier wavelength and aspect angle, diversity in either domain should lower the risk that the current RCS is near a null. This is one of the major motivations for utilizing a widely separated MIMO scheme. ${ }^{7}$

Widely separated MIMO radar has been extended to the tracking radar problem utilizing monopulse angle estimation techniques. ${ }^{8}$ In addition to any external interference such as jamming, for widely separated MIMO tracking radars any line of sight paths between transmitter and receiver may act as interference (via antenna pattern sidelobes) as well. This problem produces the need to introduce either temporal or spatial filtering. Temporal filtering (or blanking) may overlap with the expected target delay of a specific transmitter/receiver pair. The transmitter may also place nulls in the angles corresponding to the receivers which would require adaptive transmit beamforming. Similarly, the receiver may place nulls in the direction of the transmitters. Since it is more likely that the receiver will be performing adaptive array processing already to combat jamming, we consider the latter solution in this paper.

The adaptive array angle estimation problem for monopulse has been studied in the past for single-input single-output and phased array radars. ${ }^{9}$ The optimal beamforming weights have been derived for arbitrary array layouts and subarray configurations. ${ }^{10}$ The majority of these exisiting methods utilize maximum likelihood estimates where the noise is assumed to be complex Gaussian with zero mean and static covariance matrix. ${ }^{9,10}$ Unfortunately, the environment noise may not follow a Gaussian distribution. These methods also utilize the target free covariance matrix which may not be obtainable in all scenarios.

This work was supported in part by the SMART fellowship program and the University of Florida Research Foundation (UFRF) Professorships Award. William Rowe, and Jian Li are with the department of Electrical and Computer Engineering, University of Florida, Gainesville, Florida 32611, wrowe001@ufl.edu, li@dsp.ufl.edu. Jian Li is also affiliated with the IAA, Inc. Gainesville, Florida 32611. Marie Ström is with Chalmers University of Technology, Department of Signals and Systems, 41296 Göteborg, Sweden and Saab EDS, Solhusgatan 10, Kallebäcks Teknikpark, 41289 Göteborg, Sweden, marie.strom@chalmers.se Petre Stoica is with the department of Information Technology, Division of Systems and Control, Uppsala University, 75105 Uppsala, Sweden, Peter.Stoica@it.uu.se. 
In the original MIMO Monopulse tracking system proposed, an amplitude monopulse system that used the Capon beamformer was described. ${ }^{8}$ This approach may suffer in performance because the monopulse estimate utilizes symmetry between the squinted beams to generate a linear monopulse ratio. There is no guarantee that the Capon beams will provide such symmetry when interference is close to the mainbeam or number of snapshots available to estimate the target free covariance matrix is low.

In this work we examine three different beamforming approaches for adaptive amplitude monopulse. In these approaches we consider a joint design of the left and right beams for amplitude monopulse. The first method, called linearly constrained Capon (LCC), resolves the problem of a lack of symmetry when using the Capon beamformer, but it requires the target free covariance matrix. The second method introduces a norm constraint into the LCC problem. We call this method norm and linearly constrained Capon Beamformer (NLCC) and it can directly utilize the target return samples to estimate the covariance matrix. The final method, which is an extension of the adaptive matrix approach (AMA), allows for the suppression of direct path interference between transmitters and receivers by utilizing the knowledge of the scene geometry.

In the next section a brief review of monopulse estimation fundamentals will be presented to motivate the beamforming problem. This will be followed by the fundamentals of the joint beam design problem for MIMO monopulse tracking. By using these fundamentals, the LCC, NLCC, and AMA approaches will be presented. The performance will be evaluated by numerical simulations in the fourth section. Finally, we will summarize our results and conclude this paper.

We will briefly describe notation that will be used in this paper. A column vector is denoted by a boldface lower case letter $\mathbf{a}$ and a matrix is denoted by an boldface upper case $\mathbf{A}$. The target free covariance matrix will be denoted as $\mathbf{Q}$ and the covariance matrix containing the target will be denoted by $\mathbf{R}$. We also denote $(\cdot)^{H}$ as the Hermetian transpose of a vector or matrix. The operator $\operatorname{Tr}(\cdot)$ denotes a matrix trace operation. Finally we denote $\otimes$ as a Kronecker matrix product.

\section{MONOPULSE FUNDAMENTALS}

Monopulse is a computationally efficient method of measuring a target's angular error that is based on a first order Taylor expansion approximation. The concept is to generate an error signal relative to the expected target position. ${ }^{11}$ This can be achieved by either using multiple phase centers (phase monopulse) or by using squinted beams (amplitude monopulse). A difference $(\Delta)$ and a sum $(\Sigma)$ channel is formed from the multiple beams. The ratio $\Delta / \Sigma$ is called the monopulse ratio. When the beamshapes are symmetric, then the ratio is linear with respect to the true target angle in a local region. Using the Taylor expansion of the monopulse ratio, we have the following expression for estimating the target angle for uniform linear arrays (ULA):

$$
\sin (\hat{\theta})=\sin \left(\theta_{0}\right)-\gamma^{-1} \frac{\Delta}{\Sigma} .
$$

Here $\theta_{\mathrm{O}}$ is our original target angle estimate, $\hat{\theta}$ is the estimated target angle, and $\gamma$ is the slope of the monopulse ratio. ${ }^{10}$ If the monopulse ratio is not approximated by a linear function, then the angle estimate will degrade. The performance of the monopulse estimate is dependent on noise, clutter, and jammer power in the received signal as well. For this reason all monopulse beams should be designed to posses low sidelobe levels and/or jammer rejection. ${ }^{11}$

For amplitude monopulse two beams are squinted from the estimated target angle $\theta_{\mathrm{o}}$ at angles $\theta_{\mathrm{L}}$ and $\theta_{\mathrm{R}}$. The beams are squinted off by approximately half the angular resolution determined by the array size. For the data-independent amplitude monopulse, we assume that two beams $\mathbf{w}_{\mathrm{L}}$ and $\mathbf{w}_{\mathrm{R}}$ are formed using Taylor windowed standard beamforming vectors. The difference and sum channels are then defined as:

$$
\begin{aligned}
& \Delta=\left|\mathbf{w}_{\mathrm{R}}^{H} \mathbf{y}\right|-\left|\mathbf{w}_{\mathrm{L}}^{H} \mathbf{y}\right| \\
& \Sigma=\left|\mathbf{w}_{\mathrm{R}}^{H} \mathbf{y}\right|+\left|\mathbf{w}_{\mathrm{L}}^{H} \mathbf{y}\right|,
\end{aligned}
$$

where $\mathbf{y}$ is the received data vector. 
This data-independent method is computationally efficient, easy to implement and provides good performance in most scenarios. However, the data-independent approach is not robust against jammers and interference. In the widely separated MIMO scenario, the direct path between a transmitter and receiver pair could create unwanted interference even when it is arriving in the sidelobe region. We will propose adaptive amplitude beamforming methods for these MIMO systems.

\section{ADAPTIVE AMPLITUDE MONOPULSE}

An adaptive approach to the monopulse estimate was developed in 1976 for single-input single-output systems, ${ }^{9}$ and an excellent overview containing the modern theory for adaptive monopulse estimation was presented in $2006 .{ }^{10}$ In both these solutions, the ideal adaptive monopulse estimate is a phase monopulse type estimate. The estimate is based on the assumption that the noise and interference distribution is complex normal with covariance matrix $\mathbf{Q}$. The dependence on the target free covariance matrix $\mathbf{Q}$ can pose a practical problem in real world systems when target free samples are limited or not available.

Even if $\mathbf{Q}$ is available when utilizing the Capon beamformer, as proposed in the original MIMO monopulse paper, ${ }^{8}$ to generate amplitude monopulse there is no guarantee that the beams will produce a linear monopulse ratio. A loss of linearity degrades the angle estimate approximation in (1). Suppose that the linearity was not a problem, the Capon beamformer still requires $\mathbf{Q}$. The reason the Capon beamformer must use $\mathbf{Q}$ instead of target sample covariance matrix $\mathbf{R}$ is because amplitude monopulse attempts to squint two beams from $\theta_{0}$ with the assumption that our target lies between the two beam focal points. Since $\mathbf{R}$ contains the target arriving with angle between the two squinted beam peaks, the Capon beamformer will regard it as interference and try to null the target return. This can be resolved by defocusing the Capon beamformer using robust Capon beamforming (RCB) techniques. ${ }^{12}$ In the widely separated MIMO radar scenario, it would be logical to force nulls into the antenna beampattern in angles corresponding to direct paths between transmitter and receiver pairs. The adaptive matrix approach (AMA) allows for the design of beampatterns that can place desired nulls while maintaining adaptive capabilities. ${ }^{13}$

In this section we propose three methods to develop an adaptive amplitude monopulse estimate. The first method, called linearaly constrained Capon (LCC) amplitude monopulse, extends the Capon beamformer with the introduction of more linear constraints when a target free covariance matrix estimate is available. This method maintains a more linear monopulse ratio compared to the standard Capon method. The second method, called norm and linearly constrained Capon (NLCC) amplitude monopulse, extends the norm constraint RCB to include more linear constraints to help preserve the monopulse ratio. We also present a method of calculating the diagonal loading level for a given norm constraint. Finally, we present a method using the AMA for amplitude monopulse for scenarios where unwanted angles are known a priori.

\subsection{Joint Beam Design Basics}

We begin our discussion by laying out the model and a basic joint beamshape design metric. We assume that our array has a known (via calibration) steering vector $\mathbf{a}(\theta) \in \mathbb{C}^{N \times 1}$, where $N$ is the number of elements in our array and $\theta \in\left(-90^{\circ}, 90^{\circ}\right]$. We also assume that the array has been critically sampled. For amplitude monopulse we wish to jointly design two adaptive beams at angles $\theta_{\mathrm{L}}$ and $\theta_{\mathrm{R}}$. If we utilized a standard Capon beamforming cost function we would get:

$$
\begin{array}{ll}
\underset{\mathbf{w}_{\mathrm{L}}, \mathbf{w}_{\mathrm{R}}}{\operatorname{minimize}} & \mathbf{w}_{\mathrm{L}}^{H} \mathbf{Q} \mathbf{w}_{\mathbf{L}}+\mathbf{w}_{\mathrm{R}}^{H} \mathbf{Q} \mathbf{w}_{\mathrm{R}} \\
\text { subject to } & \mathbf{w}_{\mathbf{L}}^{H} \mathbf{a}\left(\theta_{\mathbf{L}}\right)=\mathbf{w}_{\mathrm{R}}^{H} \mathbf{a}\left(\theta_{\mathrm{R}}\right)=1 .
\end{array}
$$

This problem can be rewritten into the standard Capon Beamforming problem by manipulating the vectors and matrices. Let $\mathbf{w}=\left[\mathbf{w}_{\mathrm{L}}^{H} \mathbf{w}_{\mathrm{R}}^{H}\right]^{H}, \mathbf{b}=\left[\begin{array}{ll}1 & 1\end{array}\right]$, and $\tilde{\mathbf{Q}}=\mathbf{I}_{2 \times 2} \otimes \mathbf{Q}$. We then create a linear constraint matrix $\mathbf{A}$ such that:

$$
\mathbf{A}=\left[\begin{array}{ll}
\mathbf{a}\left(\theta_{\mathrm{L}}\right) & \mathbf{0}_{N \times 1} \\
\mathbf{0}_{N \times 1} & \mathbf{a}\left(\theta_{\mathrm{R}}\right)
\end{array}\right]
$$


Using $\mathbf{w}, \tilde{\mathbf{Q}}, \mathbf{b}$, and $\mathbf{A}$, we reformulate the joint beamforming problem as

$$
\begin{array}{ll}
\underset{\mathbf{w}}{\operatorname{minimize}} & \mathbf{w}^{H} \tilde{\mathbf{Q}} \mathbf{w} \\
\text { subject to } & \mathbf{w}^{H} \mathbf{A}=\mathbf{b} .
\end{array}
$$

This is a well known problem. ${ }^{14}$ A closed form solution to it is given by

$$
\mathbf{w}=\left(\tilde{\mathbf{Q}}^{-1} \mathbf{A}\right)\left(\mathbf{A}^{H} \tilde{\mathbf{Q}}^{-1} \mathbf{A}\right)^{-1} \mathbf{b}^{H} .
$$

Here the first $N$ elements of $\mathbf{w}$ correspond to $\mathbf{w}_{\mathrm{L}}$ and the last $N$ elements correspond to $\mathbf{w}_{\mathrm{R}}$. This problem would result in the standard Capon beams since the linear constraints on the beams are the same. Next we will show that the inclusion of more linear constraints into the matrix $\mathbf{A}$ and vector $\mathbf{b}$ will allow for the joint design of adaptive beams that maintain good monopulse performance.

\subsection{Adaptive Amplitude Monopulse Beamforming}

When the target free covariance matrix is available the optimal beamforming vector is given by the Capon estimator. This does not imply that the monopulse ratio formed by two squinted Capon estimators will form a good monopulse ratio. It is easy to show through numerical examples that an amplitude monopulse ratio formed with Capon beams will have non-ideal properties such as lack of linearity and non-zero response at zero angular error.

We propose to rectify such problems by the inclusion of three more linear constraints into the problem posed in (4). The first additional constraint is to force equality between the beams at the expected target position. This removes the non-zero monopulse ratio response at zero error. The next two additional constraints help promote linearity by promoting a decreasing beam slope in the measurement region. The modified problem is

$$
\begin{array}{cl}
\underset{\mathbf{w}_{\mathrm{L}}, \mathbf{w}_{\mathrm{R}}}{\operatorname{minimize}} & \mathbf{w}_{\mathrm{L}}^{H} \mathbf{Q} \mathbf{w}_{\mathbf{L}}+\mathbf{w}_{\mathrm{R}}^{H} \mathbf{Q} \mathbf{w}_{\mathrm{R}} \\
\text { subject to } & \mathbf{w}_{\mathrm{L}}^{H} \mathbf{a}\left(\theta_{\mathrm{L}}\right)=\mathbf{w}_{\mathrm{R}}^{H} \mathbf{a}\left(\theta_{\mathrm{R}}\right)=1 \\
& \mathbf{w}_{\mathrm{L}}^{H} \mathbf{a}\left(\theta_{\mathrm{o}}\right)-\mathbf{w}_{\mathrm{R}}^{H} \mathbf{a}\left(\theta_{\mathrm{o}}\right)=0 \\
& \mathbf{w}_{\mathbf{L}}^{H} \mathbf{a}\left(\theta_{\mathrm{R}}\right)=\mathbf{w}_{\mathrm{R}}^{H} \mathbf{a}\left(\theta_{\mathbf{L}}\right)=\alpha,
\end{array}
$$

where $\alpha$ is a user parameter. Our empirical findings showed that $\alpha=\sqrt{.1}$ adequately promoted a linear monopulse ratio.

Using the same approach in converting (3) to (4), we will rewrite (6) as:

$$
\begin{array}{ll}
\underset{\mathbf{w}}{\operatorname{minimize}} & \mathbf{w}^{H} \tilde{\mathbf{Q}} \mathbf{w} \\
\text { subject to } & \mathbf{w}^{H} \mathbf{A}=\mathbf{b},
\end{array}
$$

where $\mathbf{w}$ and $\tilde{\mathbf{Q}}$ are defined as in (4). We let

$$
\mathbf{A}=\left[\begin{array}{ccccc}
\mathbf{a}\left(\theta_{\mathrm{L}}\right) & \mathbf{0}_{N \times 1} & \mathbf{a}\left(\theta_{\mathrm{o}}\right) & \mathbf{a}\left(\theta_{\mathrm{R}}\right) & \mathbf{0}_{N \times 1} \\
\mathbf{0}_{N \times 1} & \mathbf{a}\left(\theta_{\mathrm{R}}\right) & -\mathbf{a}\left(\theta_{\mathrm{o}}\right) & \mathbf{0}_{N \times 1} & \mathbf{a}\left(\theta_{\mathrm{L}}\right)
\end{array}\right]
$$

and

$$
\mathbf{b}=\left[\begin{array}{lllll}
1 & 1 & 0 & \alpha & \alpha
\end{array}\right]
$$

The solution is given in (5).

This approach overcomes the problems with using just the standard Capon beamformer for amplitude monopulse estimation by providing a zero monopulse ratio at zero error and having a linear monopulse ratio. The method only works though when $\mathbf{Q}$ is available. When it is not available, the method's performance will degrade. 


\subsection{Robust Adaptive Amplitude Monopulse Beamforming}

Researchers have considered robust Capon beamforming techniques and presented several different methods. A ubiquitous theme around all of the methods is diagonal loading. The diagonal loading coefficient effectively defocuses the Capon beamformer allowing for uncertainty in target angle or array calibration. ${ }^{12}$ The monopulse problem assumes that the target angle is not known perfectly, but it should be near the expected angle $\theta_{\mathrm{o}}$. This assumption naturally poses a scenario for RCB when only the covariance matrix $\mathbf{R}$ is available.

We now propose an RCB method to provide the beamforming weights for adaptive amplitude monopulse. We utilize the same constraints as posed in (7) plus we include a norm constraint on our beamforming vector. In this method we assume that $\mathbf{Q}$ is not available and $\mathbf{R}$ must be estimated instead. First we denote $\tilde{\mathbf{R}}=\mathbf{I}_{2 \times 2} \otimes \mathbf{R}$ and then write the joint beamforming problem as

$$
\begin{array}{ll}
\underset{\mathbf{w}}{\operatorname{minimize}} & \mathbf{w}^{H} \tilde{\mathbf{R}} \mathbf{w} \\
\text { subject to } & \mathbf{w}^{H} \mathbf{A}=\mathbf{b} \\
& \|\mathbf{w}\| \leq \zeta .
\end{array}
$$

We attempt to solve (8) using Lagrange multipliers. This results in the following minimization-maximization problem

$$
\begin{array}{ll}
\max _{\boldsymbol{\mu}, \lambda} \min _{\mathbf{w}} & \mathbf{w}^{H} \tilde{\mathbf{R}} \mathbf{w}-\left(\mathbf{w}^{H} \mathbf{A}-\mathbf{b}\right) \boldsymbol{\mu}-\boldsymbol{\mu}^{H}\left(\mathbf{A}^{H} \mathbf{w}-\mathbf{b}^{H}\right)+\lambda\left(\mathbf{w}^{H} \mathbf{w}-\zeta\right) \\
\text { subject to } & \lambda \geq 0,
\end{array}
$$

where $\boldsymbol{\mu} \in \mathbb{C}^{L \times 1}$ and $L$ is the number of columns of $\mathbf{A}$. Let $g_{1}(\mathbf{w}, \boldsymbol{\mu}, \lambda)=\mathbf{w}^{H} \tilde{\mathbf{R}} \mathbf{w}-\left(\mathbf{w}^{H} \mathbf{A}-\mathbf{b}\right) \boldsymbol{\mu}-\boldsymbol{\mu}^{H}\left(\mathbf{A}^{H} \mathbf{w}-\right.$ $\left.\mathbf{b}^{H}\right)+\lambda\left(\mathbf{w}^{H} \mathbf{w}-\zeta\right)$, and by completing the square the cost function can be written as

$g_{1}(\mathbf{w}, \boldsymbol{\mu}, \lambda)=\left(\mathbf{w}-(\tilde{\mathbf{R}}+\lambda \mathbf{I})^{-1} \mathbf{A} \boldsymbol{\mu}\right)^{H}(\tilde{\mathbf{R}}+\lambda \mathbf{I})\left(\mathbf{w}-(\tilde{\mathbf{R}}+\lambda \mathbf{I})^{-1} \mathbf{A} \boldsymbol{\mu}\right)-\boldsymbol{\mu}^{H} \mathbf{A}^{H}(\tilde{\mathbf{R}}+\lambda \mathbf{I})^{-1} \mathbf{A} \boldsymbol{\mu}+\mathbf{b} \boldsymbol{\mu}+\boldsymbol{\mu}^{H} \mathbf{b}^{H}-\lambda \zeta$.

The minimizing $\mathbf{w}$ given $\boldsymbol{\mu}, \lambda$ then is

$$
\mathbf{w}_{\mathrm{opt}}=(\tilde{\mathbf{R}}+\lambda \mathbf{I})^{-1} \mathbf{A} \boldsymbol{\mu} .
$$

Then we denote $g_{2}(\boldsymbol{\mu}, \lambda)=g_{1}\left(\mathbf{w}_{\mathrm{opt}}, \boldsymbol{\mu}, \lambda\right)$ where

$$
g_{2}(\boldsymbol{\mu}, \lambda)=-\boldsymbol{\mu}^{H} \mathbf{A}^{H}(\tilde{\mathbf{R}}+\lambda \mathbf{I})^{-1} \mathbf{A} \boldsymbol{\mu}+\mathbf{b} \boldsymbol{\mu}+\boldsymbol{\mu}^{H} \mathbf{b}^{H}-\lambda \zeta .
$$

The optimal $\boldsymbol{\mu}$ given $\lambda$ can then be found by calculating the derivative of $g_{2}(\boldsymbol{\mu}, \lambda)$ with respect to $\boldsymbol{\mu}$,

$$
\frac{\partial g_{2}(\boldsymbol{\mu}, \lambda)}{\partial \boldsymbol{\mu}}=-2 \mathbf{A}^{H}(\tilde{\mathbf{R}}+\lambda \mathbf{I})^{-1} \mathbf{A} \boldsymbol{\mu}+2 \mathbf{b}^{H}=0
$$

Solving for $\boldsymbol{\mu}$ results in

$$
\boldsymbol{\mu}_{\mathrm{opt}}=\left(\mathbf{A}^{H}(\tilde{\mathbf{R}}+\lambda \mathbf{I})^{-1} \mathbf{A}\right)^{-1} \mathbf{b}^{H} .
$$

Then denoting $g_{3}(\lambda)=g_{2}\left(\boldsymbol{\mu}_{\mathrm{opt}}, \lambda\right)$ gives

$$
g_{3}(\lambda)=\mathbf{b}\left(\mathbf{A}^{H}(\tilde{\mathbf{R}}+\lambda \mathbf{I})^{-1} \mathbf{A}\right)^{-1} \mathbf{b}^{H}-\lambda \zeta .
$$

The function $g_{3}(\lambda)$ will be utilized in the calculation of the diagonal loading coefficient $\lambda$. Before considering the problem of finding $\lambda$, note that we have derived the solution for a norm constrained robust beamformer with additional linear constraints as a function of $\lambda$. Specifically that

$$
\mathbf{w}_{\text {opt }}(\lambda)=(\tilde{\mathbf{R}}+\lambda \mathbf{I})^{-1} \mathbf{A}\left(\mathbf{A}^{H}(\tilde{\mathbf{R}}+\lambda \mathbf{I})^{-1} \mathbf{A}\right)^{-1} \mathbf{b}^{H}
$$

which is just the diagonally loaded solution from the previous section. When $\lambda=0$ the $\mathbf{w}_{\mathrm{opt}}$ is similar to the LCC solution in (5). If $\lambda$ is much larger than the largest eigenvalue of $\tilde{\mathbf{R}}$ (which we denote $\sigma_{\max }$ ), then 
$\mathbf{w}_{\text {opt }} \approx \mathbf{A}\left(\mathbf{A}^{H} \mathbf{A}\right)^{-1} \mathbf{b}^{H}$ which is similar to the beamformer solution. This observation gives an approximate bound on $\lambda$ of $0 \leq \lambda \leq 10 \sigma_{\max }$.

To find $\lambda$ we utilize the eigenvalue decomposition of $\tilde{\mathbf{R}}$ to simplify the required matrix inversions and to estimate bounds for $\lambda$. This is a one time expensive computation compared to the repeated inversions of $\tilde{\mathbf{R}}$ that would be required otherwise. Let $\tilde{\mathbf{R}}=\mathbf{U} \boldsymbol{\Gamma} \mathbf{U}^{H}$ be the eigenvalue decomposition of $\tilde{\mathbf{R}}$ and let $\mathbf{Z}=\mathbf{U}^{H} \mathbf{A}$. Using the eigenvalue decomposition in (11) results in

$$
\begin{aligned}
g_{3}(\lambda) & =\mathbf{b}\left(\mathbf{A}^{H}\left(\mathbf{U} \boldsymbol{\Gamma} \mathbf{U}^{H}+\lambda \mathbf{I}\right)^{-1} \mathbf{A}\right)^{-1} \mathbf{b}^{H}-\lambda \zeta \\
& =\mathbf{b}\left(\mathbf{Z}^{H}(\boldsymbol{\Gamma}+\lambda \mathbf{I})^{-1} \mathbf{Z}\right)^{-1} \mathbf{b}^{H}-\lambda \zeta .
\end{aligned}
$$

To find the optimal parameter $\lambda$, we first calculate the partial derivative of $g_{3}(\lambda)$

$$
\frac{\partial g_{3}(\lambda)}{\partial \lambda}=\mathbf{b}\left(\mathbf{Z}^{H}(\boldsymbol{\Gamma}+\lambda \mathbf{I})^{-1} \mathbf{Z}\right)^{-1} \mathbf{Z}^{H}(\boldsymbol{\Gamma}+\lambda \mathbf{I})^{-2} \mathbf{Z}\left(\mathbf{Z}^{H}(\boldsymbol{\Gamma}+\lambda \mathbf{I})^{-1} \mathbf{Z}\right)^{-1} \mathbf{b}^{H}-\zeta .
$$

This function can be shown to be monotonically decreasing when $\lambda \geq 0$ and $\boldsymbol{\Gamma}$ is positive semi-definite (see Appendix). We define the function $h(\lambda)$ as

$$
h(\lambda)=\mathbf{b}\left(\mathbf{Z}^{H}(\boldsymbol{\Gamma}+\lambda \mathbf{I})^{-1} \mathbf{Z}\right)^{-1} \mathbf{Z}^{H}(\boldsymbol{\Gamma}+\lambda \mathbf{I})^{-2} \mathbf{Z}\left(\mathbf{Z}^{H}(\boldsymbol{\Gamma}+\lambda \mathbf{I})^{-1} \mathbf{Z}\right)^{-1} \mathbf{b}^{H} .
$$

Combining the fact that $h(\lambda)$ is monotonically decreasing and the bounds for $\lambda$ implies that $\zeta_{\max }=h(0)$ and $\zeta_{\min }=h\left(10 \sigma_{\max }\right)$. If $\zeta \leq \zeta_{\min }$ or $\zeta \geq \zeta_{\max }$, then there is no optimal $\lambda$ for that $\zeta$. Given that $\zeta$ is in bounds, if $h\left(10 \sigma_{\max }\right)>\zeta$ then $\lambda_{\text {opt }}=10 \sigma_{\max }$ using our limit on $\lambda$. Otherwise, we can calculate the optimal $\lambda$ by finding the zero crossing of $h(\lambda)-\zeta$ using a root finding algorithm such as a bisection method or Newton's method.

Table 1: Norm and Linearly Constrained Capon Beamforming for Amplitude Monopulse

Step 0 - Initialize: Calculate the eignevalue decomposition: $\tilde{\mathbf{R}}=\mathbf{U} \boldsymbol{\Gamma} \mathbf{U}^{H}$ and $\mathbf{Z}=\mathbf{U}^{H} \mathbf{A}$

Step 1: Check $h\left(10 \sigma_{\max }\right) \leq \zeta \leq h(0)$

Step 2: If $h\left(10 \sigma_{\max }\right)>\zeta$ then $\lambda=10 \sigma_{\max }$.

Step 3: Else $\lambda$ is the value that satisfies $h(\lambda)-\zeta=0$.

Step 4: $\mathbf{w}=\mathbf{U}(\boldsymbol{\Gamma}+\lambda \mathbf{I})^{-1} \mathbf{Z}\left(\mathbf{Z}^{H}(\boldsymbol{\Gamma}+\lambda \mathbf{I})^{-1} \mathbf{Z}\right)^{-1} \mathbf{b}^{H}$

When $\lambda$ is calculated then $\mathbf{w}$ can be calculated as shown in (12). However, a more computationally efficient method is using the eigenvalue decomposition as shown in Table 1. The procedure is effective when the target free covariance matrix is not available. In our simulations, $\zeta=2.5$ was found to be an effective norm-constraint that still preserved a linear monopulse ratio.

\subsection{Adaptive Matrix Approach Amplitude Monopulse Beamforming}

For a MIMO radar, direct paths between the transmitting antennas and the receiver can be regarded as interfering signals. If we have knowledge about the locations of the transmitters, then the direct paths could be cancelled out by designing beampatterns with nulls in these locations. Such a beampattern can be achieved by optimizing a weight matrix for the antenna array output, and an algorithm to do this adaptively is the AMA. ${ }^{13}$ With this method, which does not require knowledge of the target free covariance matrix, we can find a weight matrix which imposes nulls in the directions of the transmitters, as well as constraints on the sidelobe level.

For the AMA we introduce the weight matrices $\mathbf{W}_{R}$ and $\mathbf{W}_{\mathrm{L}}$ associated with the right and left beam, respectively, and let $\mathbf{T}_{\mathrm{R}}=\mathbf{W}_{\mathrm{R}} \mathbf{W}_{\mathrm{R}}^{H}$ and $\mathbf{T}_{\mathrm{L}}=\mathbf{W}_{\mathrm{L}} \mathbf{W}_{\mathrm{L}}^{H}$. Our objective is to find $\mathbf{T}_{\mathrm{R}}$ and $\mathbf{T}_{\mathrm{L}}$ which yield low sidelobe levels while maintaining the desired power and cancelling the effect of the transmitters. Following the 
methodology laid out in the original work, ${ }^{13}$ the AMA can be formulated as

$$
\begin{array}{cl}
\underset{\mathbf{T}_{\mathrm{R}}, \mathbf{T}_{\mathrm{L}}}{\operatorname{minimize}} & \operatorname{Tr}\left(\mathbf{R}\left(\mathbf{T}_{\mathrm{R}}+\mathbf{T}_{\mathrm{L}}\right)\right) \\
\text { subject to } & \mathbf{a}\left(\theta_{\mathrm{R}}\right)^{H} \mathbf{T}_{\mathrm{R}} \mathbf{a}\left(\theta_{\mathrm{R}}\right)=\mathbf{a}\left(\theta_{\mathrm{L}}\right)^{H} \mathbf{T}_{\mathrm{L}} \mathbf{a}\left(\theta_{\mathrm{L}}\right)=1 \\
& \mathbf{a}\left(\theta_{\mathrm{o}}\right)^{H}\left(\mathbf{T}_{\mathrm{R}}-\mathbf{T}_{\mathrm{L}}\right) \mathbf{a}\left(\theta_{\mathrm{o}}\right)=0 \\
& \mathbf{a}\left(\theta_{\mathrm{L}}\right)^{H} \mathbf{T}_{\mathrm{R}} \mathbf{a}\left(\theta_{\mathrm{L}}\right) \leq \alpha \\
& \mathbf{a}\left(\theta_{\mathrm{R}}\right)^{H} \mathbf{T}_{\mathrm{L}} \mathbf{a}\left(\theta_{\mathrm{R}}\right) \leq \alpha \\
& \mathbf{a}\left(\mu_{\mathrm{R}}\right)^{H} \mathbf{T}_{\mathrm{R}} \mathbf{a}\left(\mu_{\mathrm{R}}\right) \leq \xi, \quad \mu_{\mathrm{R}} \in \Phi_{\mathrm{R}} \\
& \mathbf{a}\left(\mu_{\mathrm{L}}\right)^{H} \mathbf{T}_{\mathrm{L}} \mathbf{a}\left(\mu_{\mathrm{L}}\right) \leq \xi, \quad \mu_{\mathrm{L}} \in \Phi_{\mathrm{L}} \\
& \mathbf{a}\left(\mu_{\mathrm{m}}\right)^{H}\left(\mathbf{T}_{\mathrm{R}}+\mathbf{T}_{\mathrm{L}}\right) \mathbf{a}\left(\mu_{\mathrm{m}}\right) \leq \beta, \quad \mu_{\mathrm{m}} \in \Phi_{\mathrm{m}} \\
& \mathbf{T}_{\mathrm{R}} \geq 0, \mathbf{T}_{\mathrm{L}} \geq 0 .
\end{array}
$$

In (15), the first constraint is for equivalent gain in the boresight direction of the beams, the next three constraints emphasize a linear monopulse ratio in the measurement region. Similar to LCC and NLCC, $\alpha$ is a user parameter. The fifth and sixth constraint restrict the sidelobe level to $\xi$, where $\Phi_{\mathrm{R}}$ and $\Phi_{\mathrm{L}}$ are the sidelobe regions for the right and left beam, respectively. The location knowledge of the transmitters is introduced in $\Phi_{\mathrm{m}}$, where $\beta$ controls the desired depth of the nulls in the beampattern. To solve the problem, a semidefinite programming solver such as CVX can be used. ${ }^{15}$ However, it is worth noting is that if the design parameters $\xi$, $\alpha$, and $\beta$ are set too small the problem may become infeasible. For our examples we found that $\beta=0.01, \xi=10^{-4}$, and $\alpha=0.5$ produced good results.

\section{NUMERICAL EXAMPLES}

In the following numerical example we investigate one receiver in a widely separated MIMO radar system. The receiver consists of an 11 element ULA with element spacing of $\lambda / 2$. The number of snapshots available for estimation of $\mathbf{Q}$ and $\mathbf{R}$ is 22 (twice the number of elements). The return $\mathbf{y}$ is corrupted with additive zero mean Complex Gaussian noise with covariance matrix $\sigma^{2} \mathbf{I}$. The noise power $\sigma^{2}$ is set such that the signal-to-noise power ratio is $10 \mathrm{~dB}$.

The methods are evaluated based on their ability to accurately estimate the true target angle. This is achieved by first estimating the covariance matrices $\mathbf{R}$ and $\mathbf{Q}$ as appropriate. Second the left and right beamformer vectors $\mathbf{w}_{\mathrm{L}}$ and $\mathbf{w}_{\mathrm{R}}$ are generated. Using these beams the monopulse ratio slope $\gamma$ is estimated in the expected target region. The target is assumed to be coherent over the 22 snapshots and $\overline{\mathbf{y}}=1 / N \sum_{i}^{22} \mathbf{y}_{\mathrm{i}}$. Then the sum and difference channel measurements are calculated using $\overline{\mathbf{y}}, \mathbf{w}_{\mathrm{L}}$ and $\mathbf{w}_{\mathrm{R}}$ in (3). For the AMA method, the left and right channel estimate is done using $\operatorname{Tr}\left(\mathbf{R} \mathbf{W}_{\mathrm{L}}\right)$ and $\operatorname{Tr}\left(\mathbf{R} \mathbf{W}_{\mathbf{R}}\right)$. Finally, the angle estimate is made by solving (1) for $\hat{\theta}$. The squared absolute error is measured relative to the true incoming target position.

The methods are evaluated over 4 different scenarios. The first scenario is the noise only case which we use as a baseline for comparison. The second scenario is when white noise jammers are present as well as noise. The jammers are $20 \mathrm{~dB}$ stronger than the return signal and jamming signals are arriving at $-35^{\circ}$ and $15^{\circ}$. The third scenario includes the same jammers and noise, but the target free covariance matrix is not available. In the fourth scenario a direct path exists between one of the transmitters and the receiver at $-65^{\circ}$ with energy 20 $\mathrm{dB}$ greater than the target return. There is only one jammer at $15^{\circ}$ with energy $20 \mathrm{~dB}$ greater than the target and the SNR is $10 \mathrm{~dB}$. The angle for the direct path is known before hand for the AMA method.

For each scenario 100 Monte Carlo trials were performed and the squared absolute error for the angular estimate was measured. The monopulse ratios for each method and the beamshapes were also recorded for each trial. In a Monte Carlo trial, the expected target angle of arrival was $0^{\circ}$, but the actual target angle was uniformly distributed between $\pm 5^{\circ}$. For each trial an independent set of noise is generated as well.

For each methods the beams were squinted $5^{\circ}$ since the array resolution was approximately $10^{\circ}$. We use the parameter $\alpha=\sqrt{.1}$ for LCC and NLCC, but 0.5 for AMA. The norm constraint used for NLCC was $\zeta=2.5$ and $\zeta$ was always within tolerance. For AMA the sidelobe level parameter $\xi=0.01$ and the direct path rejection 
level was $\beta=10^{-4}$ and a feasible solution was found for every trial. The non-adaptive method utilized a Taylor window with $20 \mathrm{~dB}$ sidelobe level and parameter $\bar{n}=6$.

Figure 1 shows the box plots of the absolute angle error for the Monte Carlo simulations. The boxes are all cropped to $3^{\circ}$ for visibility and because the best methods for each scenario are all contained in that region. Figure 2 is the data independent beamshape used for every scenario and the resulting monopulse ratio. Figures 3-6 shows 20 samples from each Monte Carlo run of the beamshapes and the resulting monopulse ratio for each method.

In the first scenario, the data independent approach is the most pragmatic method due to the computational simplicity and good performance as seen in Figure 1(a). The Capon amplitude monopulse in Figure 3(b) does not maintain a linear monopulse ratio in this scenario, while all other methods maintain good linearity in the desired region. This lack of linearity makes the angle estimate a poor one as can be seen from the error. The proposed methods in Figures 3(c)-(e) all maintain good linearity while maintaining error similar to the non-adaptive approach.

The second scenario introduces jammers into the problem. In Figure 1(b), it is clear that the error for the data independent approach degrades significantly as expected. The other methods error remains similar to scenario 1. The Capon method and the linear constraint amplitude monopulse estimate both have access to a target free covariance matrix estimate. The NLCC and AMA approaches use the covariance matrix estimated from the returned data. The Capon amplitude monopulse beamformer still does not have a very linear monopulse ratio as can be seen in Figure 4(b). The proposed adaptive methods all retain good linearity as shown in Figures $4(\mathrm{~b})-(\mathrm{d})$.

The third scenario is when the target free covariance matrix is not available. The Capon and proposed LCC monopulse beamformer both degrade as can be seen in Figure 1(c). Both the LCC and Capon methods monopulse ratios lose their linearity as see in Figures 5(a)-(b), but the LCC method degrades less. Since the NLCC and AMA methods are designed to use the covariance matrix estimate containing the target their error remains similar to the previous 2 scenarios.

The final scenario is when a direct path is present between the transmitter and receiver. The Capon and linearly constrained amplitude monopulse method both have access to the target free covariance matrix estimate again. The AMA method places a null in a 0.5 degree region around the known angle. Figure 1.(d) shows that direct path energy spoils all the other methods except the AMA. However, the AMA error has approximately doubled in this scenario. It can be seen from Figure 6(d) that a null is placed in the region of the direct path energy.

\section{CONCLUSIONS}

The use of widely separated MIMO radar systems for target tracking is useful since it mitigates target glint. In the MIMO scenario the standard monopulse approaches may not be enough and it poses a new beamforming problem. The results presented in this work suggest that the standard Capon method should not be used because there is no guarantee of a linear monopulse response. The linearly constrained adaptive amplitude monopulse method proposed here extends the Capon method and is presented in a closed form solution. However in scenarios where the target-free covariance matrix is not available, the results suggest the use of the norm constrained beamformer or the adaptive matrix approach. When nulls are required due to the geometry between the transmitter and the receivers, the adaptive matrix approach is recommended. 


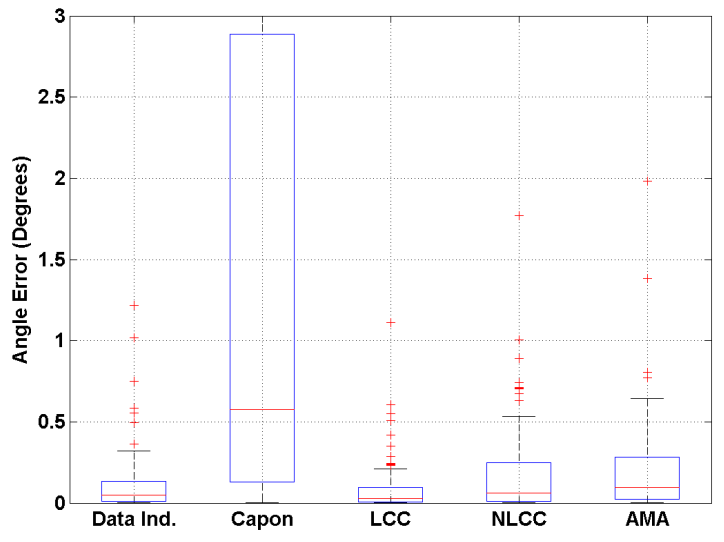

(a) Scenario 1: Gaussian Noise $10 \mathrm{~dB}$

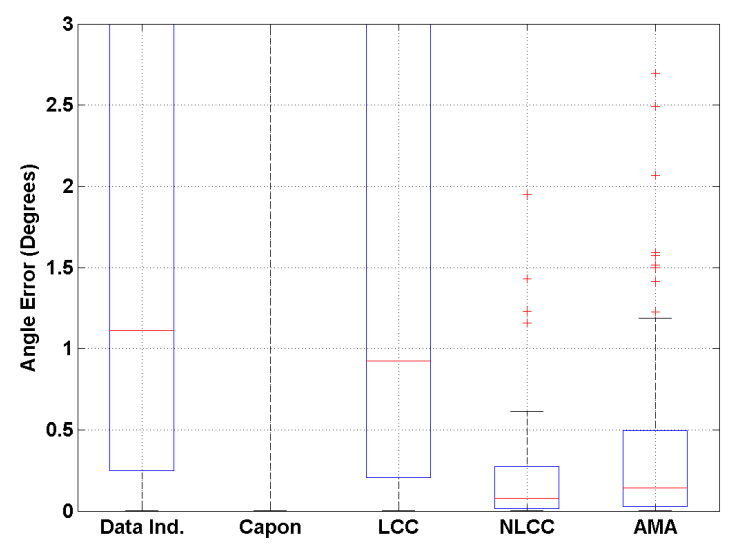

(c) Scenario 3: Target Free Covariance Matrix Not Available

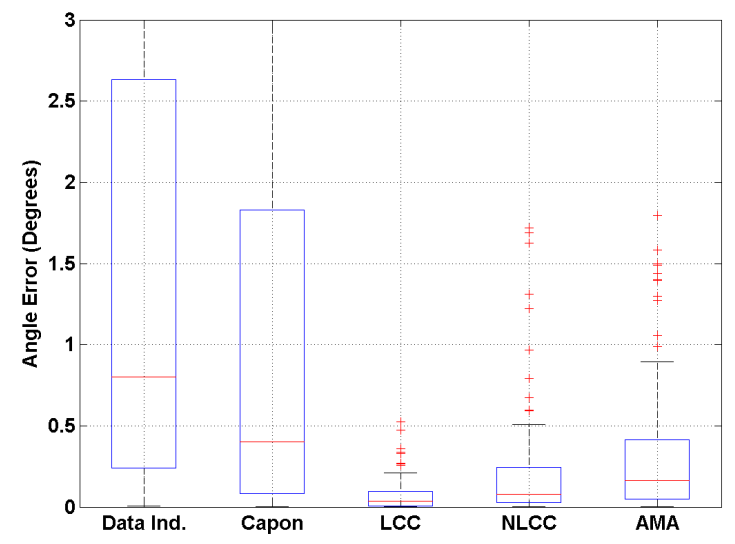

(b) Scenario 2: $10 \mathrm{~dB}$ Noise and $20 \mathrm{~dB}$ Jammers

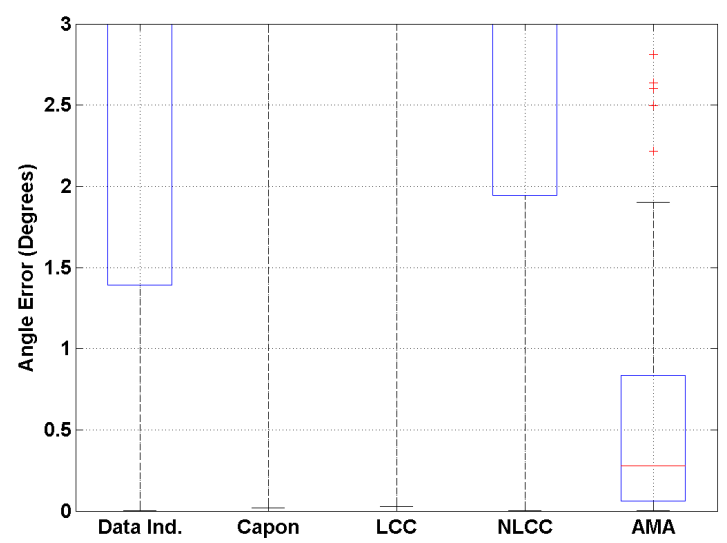

(d) Scenario 4: Direct Path Energy Present Figure 1: Box plots for Absolute Angular Error Squared for 100 Monte Carlo Trials
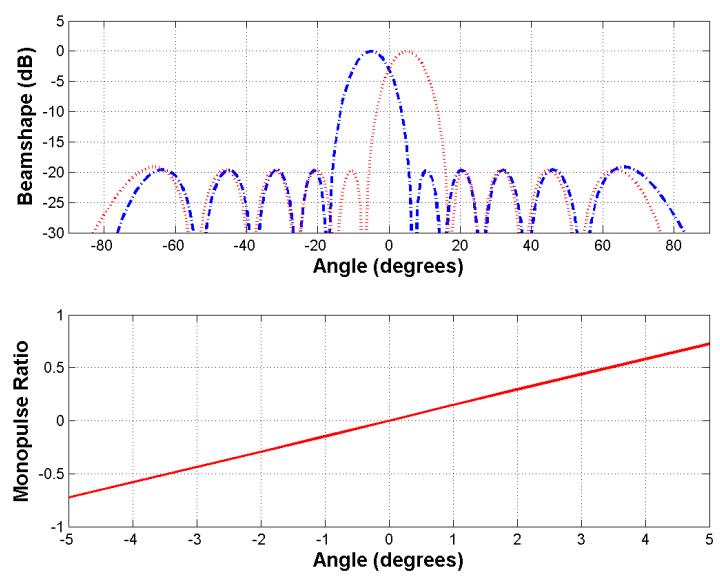

Figure 2: Data Independent Amplitude Monopulse 

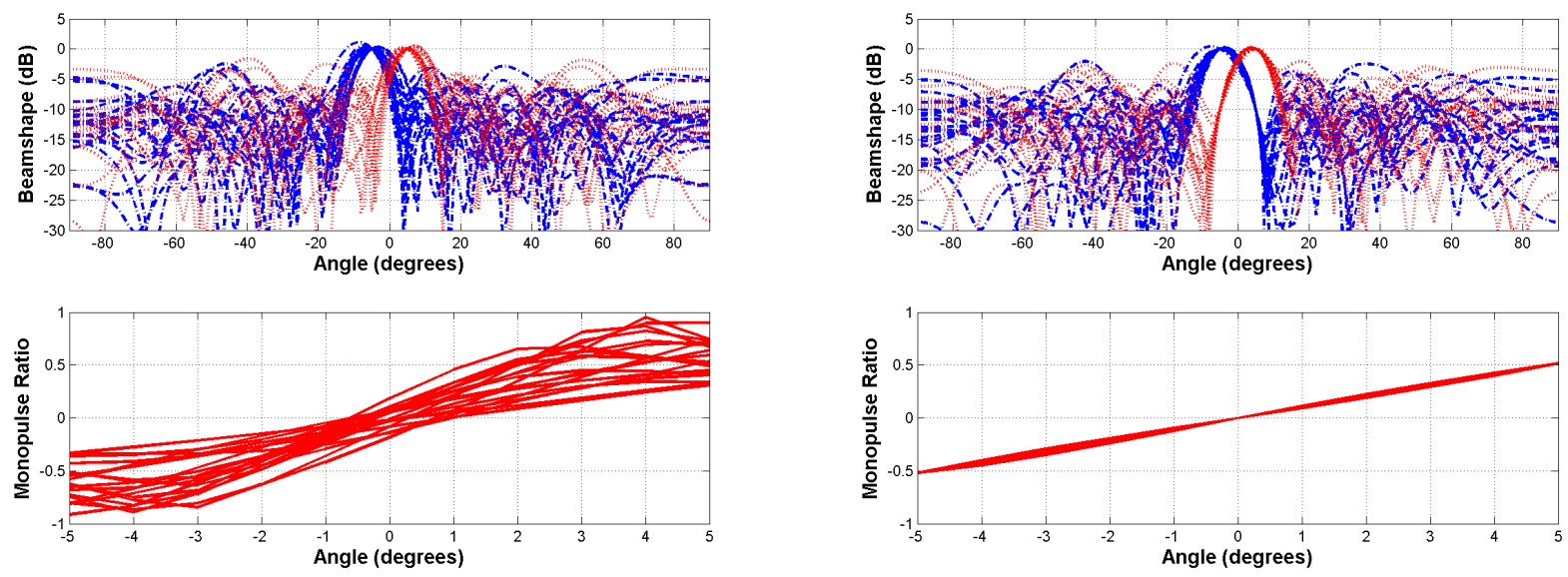

(a) Capon Amplitude Monopulse

(b) Linear Constrained Adaptive Amplitude Monopulse
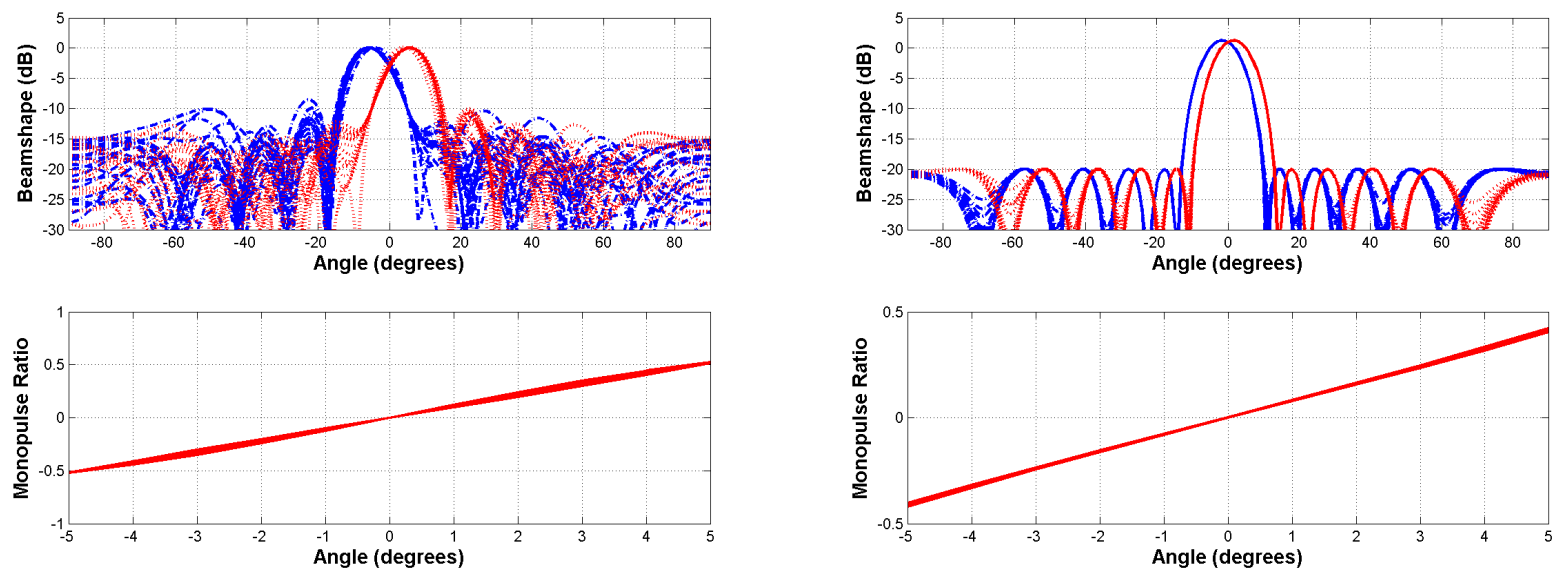

(c) Norm Constrained Adaptive Amplitude Monopulse

(d) Adaptive Matrix Approach Amplitude Monopulse Figure 3: Scenario 1 Beamshapes and Monopulse Ratios 

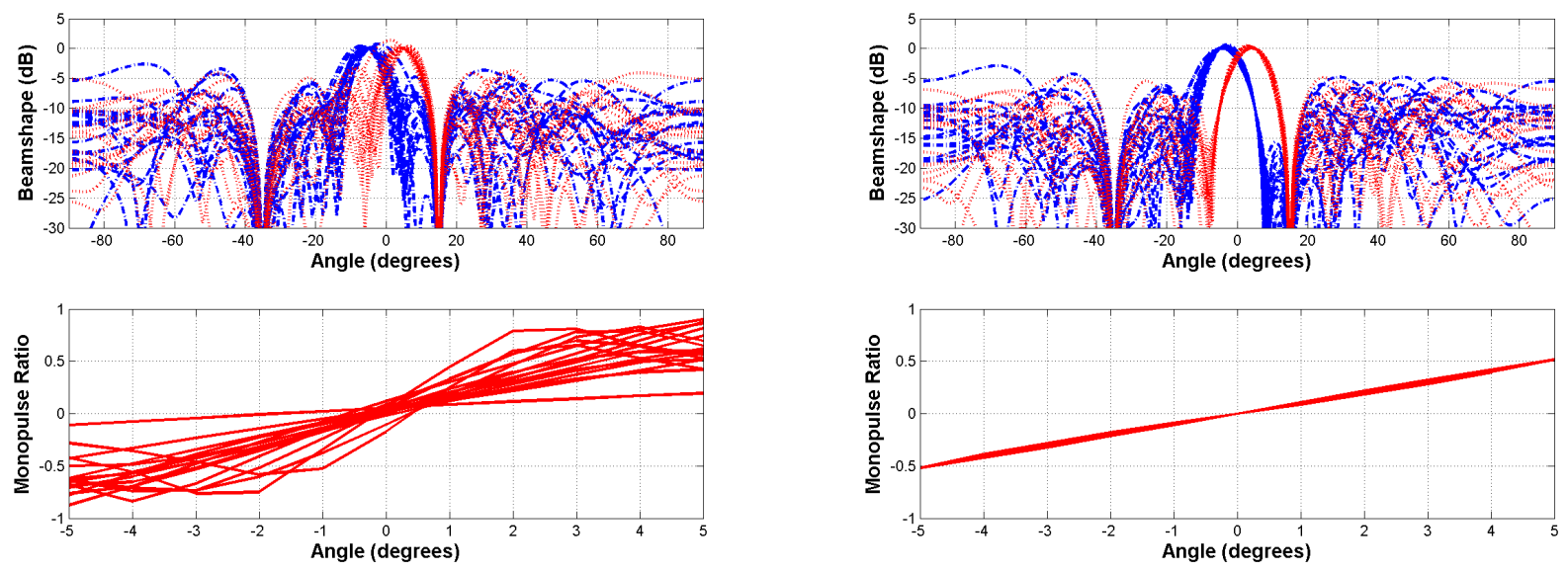

(a) Capon Amplitude Monopulse

(b) Linear Constrained Adaptive Amplitude Monopulse
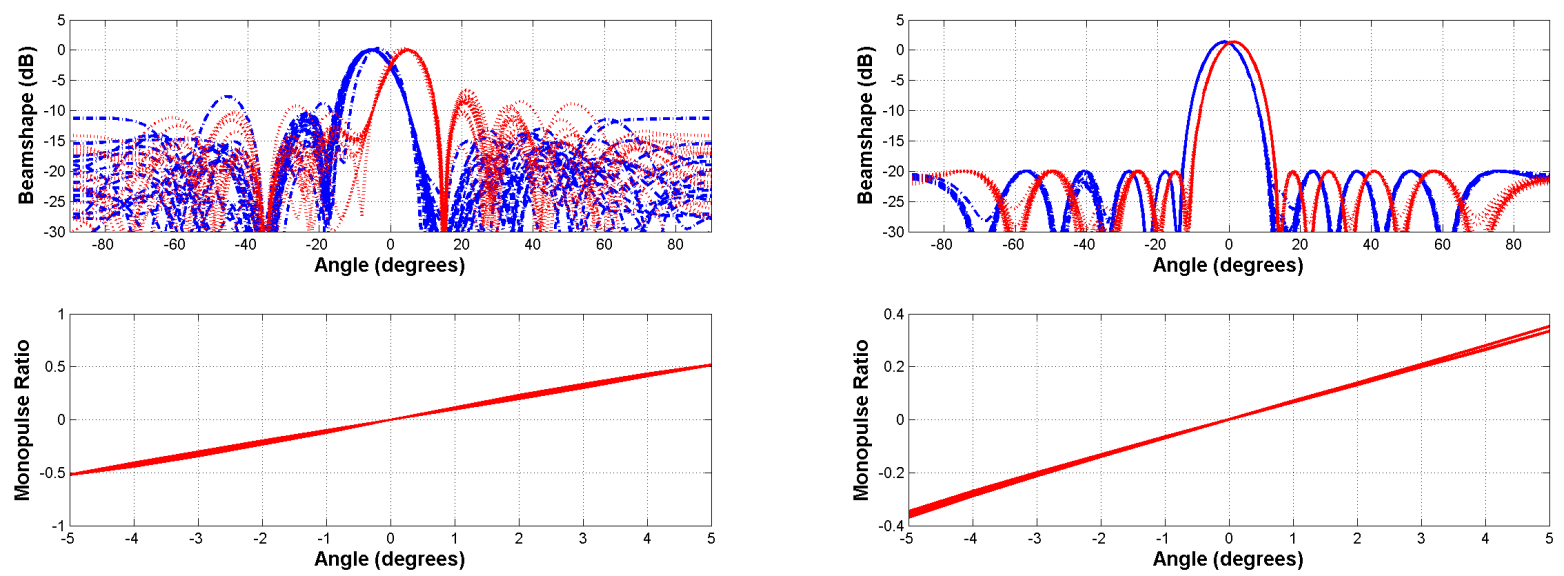

(c) Norm Constrained Adaptive Amplitude Monopulse

(d) Adaptive Matrix Approach Amplitude Monopulse Figure 4: Scenario 2 Beamshapes and Monopulse Ratios 

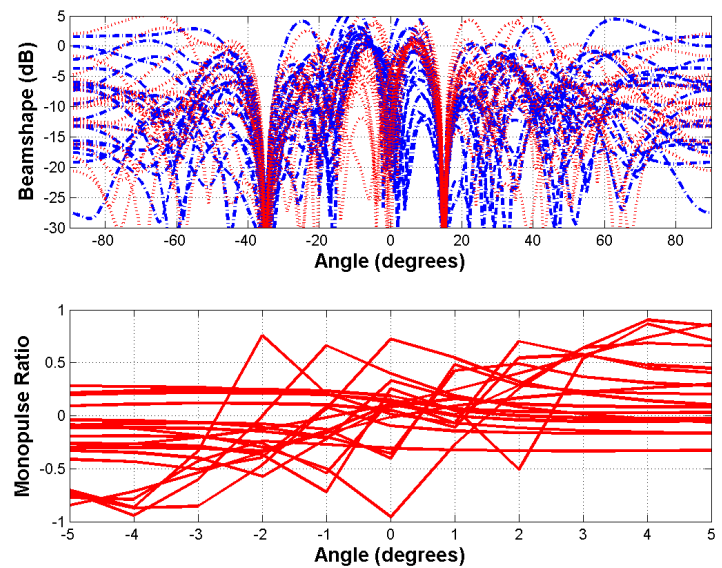

(a) Capon Amplitude Monopulse
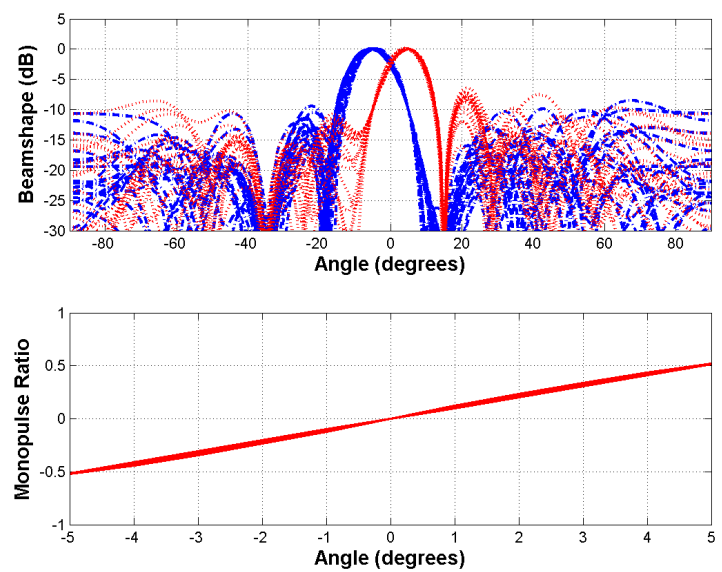

(c) Norm Constrained Adaptive Amplitude Monopulse
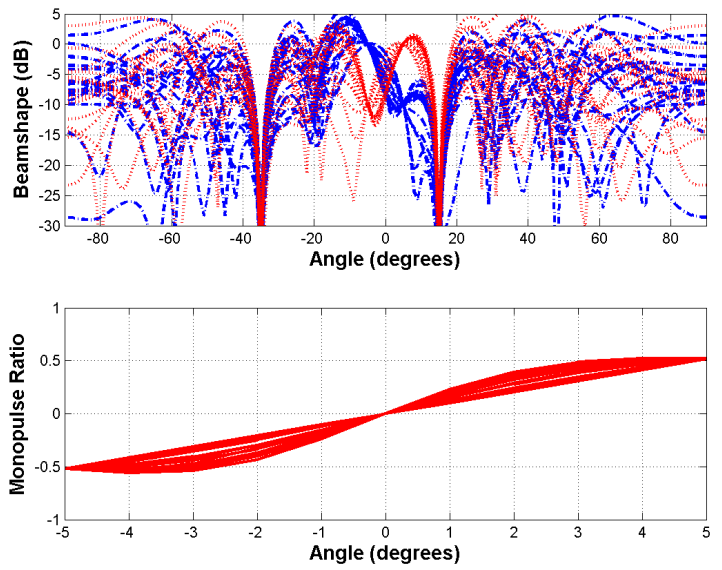

(b) Linear Constrained Adaptive Amplitude Monopulse
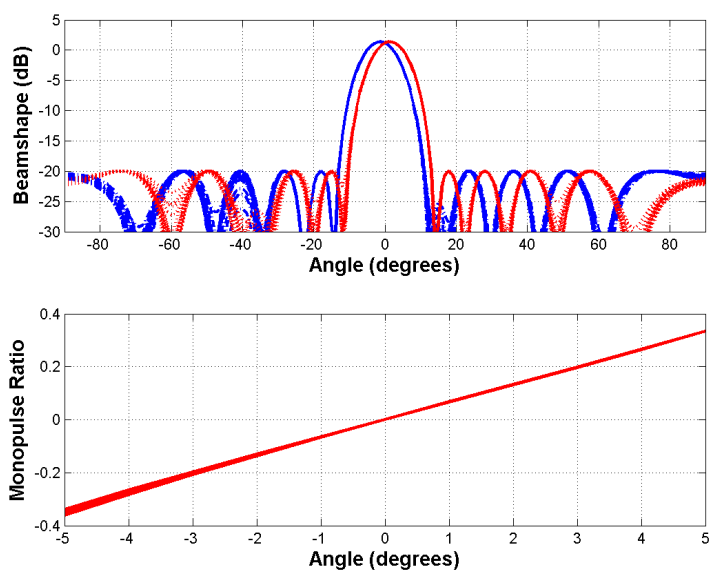

(d) Adaptive Matrix Approach Amplitude Monopulse Figure 5: Scenario 3 Beamshapes and Monopulse Ratios 

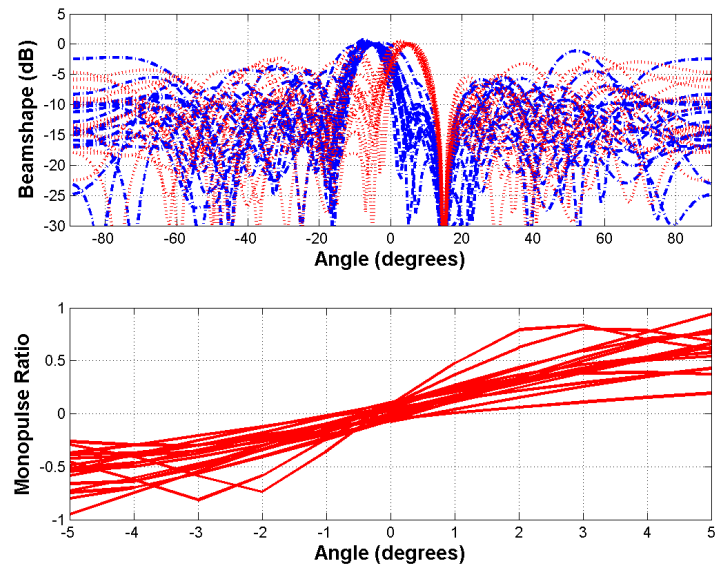

(a) Capon Amplitude Monopulse
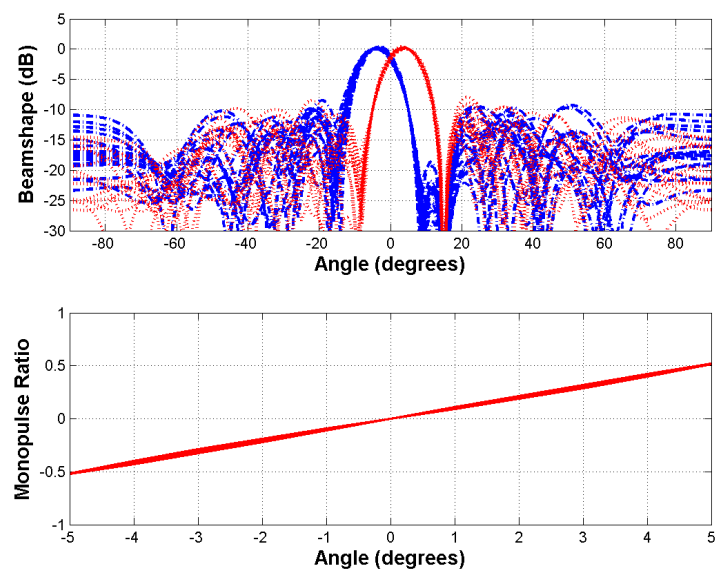

(c) Norm Constrained Adaptive Amplitude Monopulse
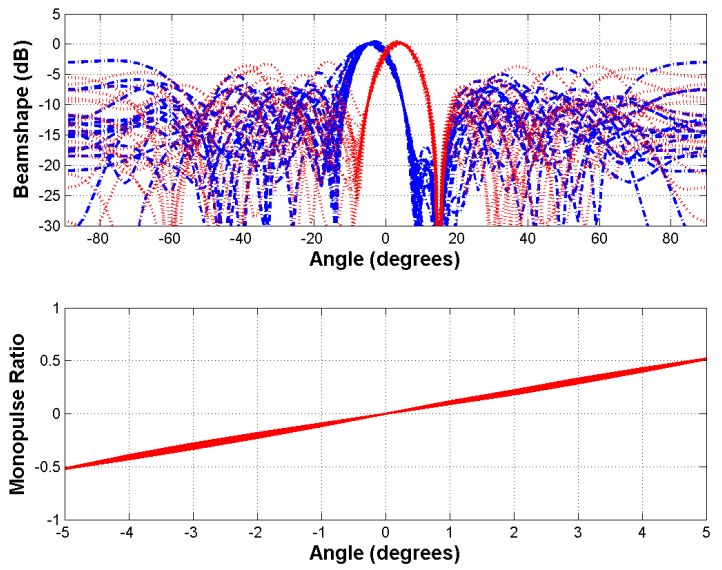

(b) Linear Constrained Adaptive Amplitude Monopulse
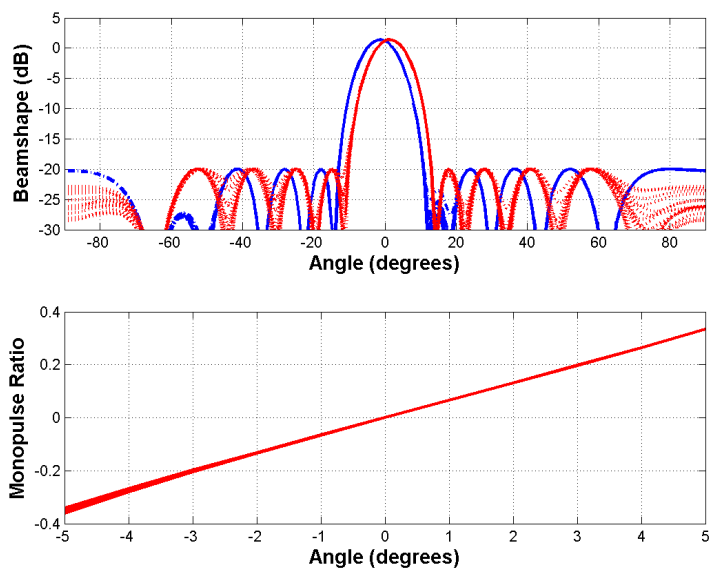

(d) Adaptive Matrix Approach Amplitude Monopulse Figure 6: Scenario 4 Beamshapes and Monopulse Ratios 


\section{APPENDIX}

It will now be shown that the function $h(\lambda)$ is a monotonically decreasing function when $\lambda>0$ and when $\boldsymbol{\Gamma}$ is positive semi-definite. Denoting $\mathbf{F}=(\boldsymbol{\Gamma}+\lambda \mathbf{I})$ and using the following derivatives:

$$
\begin{aligned}
\frac{\partial \mathbf{F}}{\partial \lambda} & =\mathbf{I}, \\
\frac{\partial \mathbf{F}^{-1}}{\partial \lambda} & =-\mathbf{F}^{-2}, \\
\frac{\partial \mathbf{F}^{-2}}{\partial \lambda} & =-2 \mathbf{F}^{-3}, \\
\frac{\partial\left(\mathbf{Z}^{H} \mathbf{F}^{-1} \mathbf{Z}\right)^{-1}}{\partial \lambda} & =\left(\mathbf{Z}^{H} \mathbf{F}^{-1} \mathbf{Z}\right)^{-1} \mathbf{Z}^{H} \mathbf{F}^{-2} \mathbf{Z}\left(\mathbf{Z}^{H} \mathbf{F}^{-1} \mathbf{Z}\right)^{-1}, \\
\frac{\partial\left(\mathbf{Z}^{H} \mathbf{F}^{-2} \mathbf{Z}\right)}{\partial \lambda} & =-2 \mathbf{Z}^{H} \mathbf{F}^{-3} \mathbf{Z} .
\end{aligned}
$$

The function $h(\lambda)$ is redefined here for clarity as

$$
h(\lambda)=\mathbf{b}\left(\mathbf{Z}^{H} \mathbf{F}^{-1} \mathbf{Z}\right)^{-1} \mathbf{Z}^{H} \mathbf{F}^{-2} \mathbf{Z}\left(\mathbf{Z}^{H} \mathbf{F}^{-1} \mathbf{Z}\right)^{-1} \mathbf{b}^{H} .
$$

Using the previously calculated derivatives, $h^{\prime}(\lambda)$ can be calculated as

$$
h^{\prime}(\lambda)=-2 \mathbf{b}\left(\mathbf{Z}^{H} \mathbf{F}^{-1} \mathbf{Z}\right)^{-1}\left\{\mathbf{Z}^{H} \mathbf{F}^{-3} \mathbf{Z}-\mathbf{Z}^{H} \mathbf{F}^{-2} \mathbf{Z}\left(\mathbf{Z}^{H} \mathbf{F}^{-1} \mathbf{Z}\right)^{-1} \mathbf{Z}^{H} \mathbf{F}^{-2} \mathbf{Z}\right\}\left(\mathbf{Z}^{H} \mathbf{F}^{-1} \mathbf{Z}\right)^{-1} \mathbf{b}^{H} .
$$

Then by factoring we can rewrite $h^{\prime}(\lambda)$ as

$$
h^{\prime}(\lambda)=-2 \mathbf{b}\left(\mathbf{Z}^{H} \mathbf{F}^{-1} \mathbf{Z}\right)^{-1} \mathbf{Z}^{H} \mathbf{F}^{-3 / 2}\left\{\mathbf{I}-\mathbf{F}^{-1 / 2} \mathbf{Z}\left(\mathbf{Z}^{H} \mathbf{F}^{-1 / 2} \mathbf{F}^{-1 / 2} \mathbf{Z}\right)^{-1} \mathbf{Z}^{H} \mathbf{F}^{-1 / 2}\right\} \mathbf{F}^{-3 / 2} \mathbf{Z}\left(\mathbf{Z}^{H} \mathbf{F}^{-1} \mathbf{Z}\right)^{-1} \mathbf{b}^{H} .
$$

The first section $\mathbf{b}\left(\mathbf{Z}^{H} \mathbf{F}^{-1} \mathbf{Z}\right)^{-1} \mathbf{Z}^{H} \mathbf{F}^{-3 / 2}$ is a row vector and the last section is its conjugate transpose. The matrix $\mathbf{I}-\mathbf{F}^{-1 / 2} \mathbf{Z}\left(\mathbf{Z}^{H} \mathbf{F}^{-1 / 2} \mathbf{F}^{-1 / 2} \mathbf{Z}\right)^{-1} \mathbf{Z}^{H} \mathbf{F}^{-1 / 2}$ is the projection matrix onto the space orthogonal to $\mathbf{F}^{-1 / 2} \mathbf{Z}$. It is well known that any projection matrix is positive definite. This implies that $h^{\prime}(\lambda) \leq 0$ for $\lambda>0$ and $\mathbf{F}$ positive semi-definite.

\section{REFERENCES}

[1] Skolnik, M., [Introduction to Radar Systems, Second Edition], McGraw-Hill, New York, NY (1981).

[2] Dunn, J., Howard, D., and King, A., "Phenomena of scintillation noise in radar-tracking systems," Proceedings of the IRE 47, 855-863 (May 1959).

[3] Skolnik, M. I., [Radar Handbook, Third Edition], McGraw-Hill, New York, NY (2008).

[4] Borden, B., "Requirements for optimal glint reduction by diversity methods," IEEE Transactions on Aerospace and Electronic Systems 30, 1108-1114 (Oct 1994).

[5] Yin, H. and Huang, P., "Further comparison between two concepts of radar target glint," IEEE Transactions on Aerospace and Electronic Systems 44, 372-380 (Jan 2008).

[6] Sims, R. and Graf, E., "The reduction of glint by diversity techniques," IEEE Transactions on Antennas and Propogation 19, 462-468 (1971 1971).

[7] Haimovich, A. H., Blum, R. S., and Cimini, L. J., "MIMO radar with widely separated antennas," IEEE Signal Processing Magazine 25, 116-129 (Jan 2008).

[8] Gogineni, S. and Nehorai, A., "Monopulse mimo radar for target tracking," IEEE Transactions on Aerospace and Electronic Systems 47, 755-768 (Jan 2011).

[9] Davis, R., Brennan, L., and Reed, I., "Angle estimation with adaptive arrays in external noise fields," IEEE Transactions on Aerospace and Electronic Systems AES-, 179-186 (March 1976).

[10] Nickel, U., "Overview of generalized monopulse estimation," IEEE Aerospace and Electronic Systems Magazine 21, 27-56 (June 2006).

[11] Barton, D. K., [Modern Radar System Analysis], Artech House Inc., Norward, MA (1988). 
[12] Li, J. and Stoica, P., [Robust Adaptive Beamforming], John Wiley \& Sons, New York, NY (2005).

[13] Li, J., Xie, Y., Stoica, P., Zheng, X., and Ward, J., "Beampattern synthesis via a matrix approach for signal power estimation," IEEE Transactions on Signal Processing 55, 5643-5657 (December 2007).

[14] Stoica, P. and Moses, R. L., [Spectral Analysis of Signals], Prentice-Hall, Upper Saddle River, NJ (2005).

[15] CVX Research, I., "Cvx: Matlab software for disciplined convex programming, version 2.0 beta," (March 2013). 